\title{
Real Quadratic Fields With Class Numbers Divisible by Five
}

\author{
By Charles J. Parry
}

\begin{abstract}
Conditions are given for a real quadratic field to have class number divisible by five. If 5 does not divide $m$, then a necessary condition for 5 to divide the class number of the real quadratic field with conductor $m$ or $5 m$ is that 5 divide the class number of a certain cyclic biquadratic field with conductor $5 \mathrm{~m}$. Conversely, if 5 divides the class number of the cyclic field, then either one of the quadratic fields has class number divisible by 5 or one of their fundamental units satisfies a certain congruence condition modulo 25 .
\end{abstract}

1. Introduction. While a necessary and sufficient condition for 3 to divide the class number of a real quadratic field has been given by Herz [3], no similar condition seems to exist for 5. In this article, we will extend the methods of Herz to obtain such a result. Although Weinberger [9] and Yamatoto [10] have proved the existence of infinitely many real quadratic fields with class number divisible by any integer $n$, their results are quite different from those of Herz and those of this article.

Certainly 5 divides the class number of one of the quadratic fields $k_{1}=Q(\sqrt{m})$ or $k_{2}=Q(\sqrt{5 m})$ if and only if 5 divides the class number of their biquadratic compositum $K_{1}$. We show if 5 divides the class number of $K_{1}$ then 5 divides the class number of a certain imaginary cyclic biquadratic field $K_{2}$ with conductor $5 D$, where $D$ is the discriminant of $k_{1}$. Conversely, if 5 divides the class number of $K_{2}$, then either 5 divides the class number of $K_{1}$ or one of three congruence conditions holds modulo 5 or 25 on the fundamental units of $k_{1}$ or $k_{2}$.

\section{Notation.}

$\zeta=e^{2 \pi i / 5}$.

$m$ : a square free positive rational integer with $(5, m)=1$.

$Q$ : the field of rational numbers.

$k_{1}=Q(\sqrt{m})$.

$k_{2}=Q(\sqrt{5 m})$.

$k_{3}=Q(\sqrt{5})$.

$L=Q(\zeta, \sqrt{m})$.

$K_{1}=Q(\sqrt{5}, \sqrt{m})$.

$K_{2}=Q(\sqrt{-10 m+2 m \sqrt{ } 5})$ : cyclic biquadratic subfield of $L$.

$K_{3}=Q(\zeta)$.

$D=$ discriminant of the field $k_{1}$.

$h=$ class number of $L$. 
$h_{i}(i=1,2,3)$ : class number of $K_{i}$.

$h_{i}^{*}(i=1,2,3)$ : class number of $k_{i}$.

$\hat{E}:$ the group of units of $L$.

$\hat{e}$ : the subgroup of $\hat{E}$ generated by the units of fields $K_{i}(i=1,2,3)$.

$\hat{\epsilon}$ : the subgroup of $\hat{e}$ generated by the units of the fields $k_{i}(i=1,2,3)$.

$Q_{0}=(\hat{E}: \hat{e})$.

$Q_{1}=(\hat{e}: \hat{\epsilon})$.

$\epsilon_{i}(i=1,2,3)$ : the fundamental unit of the field $k_{i}$.

\section{Class Number Relations.}

THEOREM 1. $2 h=h_{1} h_{2}$.

Proof. Since the Galois group of $L / k_{3}$ is bicyclic of order 4 , it follows from Theorem 5.5.1 of Walter [8] that $2 h h_{3}^{*}=Q_{0} h_{1} h_{2} h_{3}$. However, it is well known that $h_{3}=h_{3}^{*}=1$.

To complete the proof we need to show $Q_{0}=1$. If $E \in \hat{E}$, Theorem 1 of Parry [7] shows

$$
E^{2}= \pm \zeta e= \pm \zeta^{6} e
$$

where $e \in K_{1}$. Thus,

$$
\left(E / \zeta^{3}\right)^{2}= \pm e .
$$

If $e_{1}=E / \zeta^{3} \notin K_{1}$, then $L=K_{1}\left(e_{1}\right)=K_{1}(\sqrt{ \pm e})$ so only the prime divisors of 2 in $K_{1}$ could ramify in $L$. However, the prime divisors of 5 in $K_{1}$ ramify in $L$. Thus, $e_{1}$ $\in K_{1}$ and so $E=\zeta^{3} e_{1} \in \hat{e}$. Hence, $\hat{E} \subset \hat{e}$ so $Q_{0}=1$.

THEOREM 2. $4 h_{1}=Q_{1} h_{1}^{*} h_{2}^{*}$ with $Q_{1}=1$ or 2 .

Proof. Immediate from Satz 1 of Kubota [5] and Satz 11 of Kuroda [6] since $h_{3}^{*}=1$ and the fundamental unit of $k_{3}$ has norm -1 .

Corollary 3. $8 h=Q_{1} h_{1}^{*} h_{2}^{*} h_{2}$.

\section{Class Number Divisibility.}

Lemma 4. If $5 \mid h_{1}$, then $5 \mid h_{2}$.

Proof. If $M / K_{1}$ is cyclic of degree 5 , then $M(\zeta) / K_{1}$ is cyclic of degree 10 . A generator $\sigma$ of the Galois group $G\left(M / K_{1}\right)$ can be extended to an element of $G\left(M(\zeta) / K_{1}\right)$ by setting $\zeta^{\sigma}=\zeta$. Hilbert's Theorem 90 gives an element $\alpha \in M(\zeta)$ satisfying $\alpha^{\sigma-1}=$ $\zeta$. Moreover, $\alpha$ is uniquely determined up to multiplication by $\beta \in L$.

Let $\rho$ be the unique element of $G\left(M(\zeta) / K_{1}\right)$ which has order 2 and define quantities $\theta, a$ and $e$ by $\theta=\alpha+\alpha^{\rho}, a=\alpha^{1+\rho}$ and $e=\alpha^{4-\rho}+\alpha^{4 \rho-1}$. Now $a, e \in K_{1}$, $\theta \in M, M=K_{1}(\theta)$ and $\theta^{5}-5 a \theta^{3}+5 a^{2} \theta-a e=0$. Since $M / k_{3}$ is dihedral, the nontrivial automorphism of $K_{1} / k_{3}$ can be extended to an automorphism $\tau$ of $M(\zeta) / k_{3}$ satisfying the following properties:

$$
\zeta^{\tau}=\zeta^{4}, \quad \tau^{2}=1, \quad \rho \tau=\tau \rho, \quad \tau \sigma=\sigma^{4} \tau .
$$

If $\beta=\alpha^{\tau-1}$ then

$$
\beta^{\sigma}=\left(\alpha^{\tau-1}\right)^{\sigma}=\alpha^{\sigma^{4} \tau-\sigma}=\left(\zeta^{4} \alpha\right)^{\tau} /(\zeta \alpha)=\zeta \alpha^{\tau} / \zeta a=\alpha^{\tau-1}=\beta,
$$


so that $\beta \in L$. Replace $\alpha$ with $(1+\beta) \alpha$ if $\beta \neq-1$ and with $\left(\zeta-\zeta^{4}\right) \alpha$ if $\beta=-1$. This gives $\alpha=\alpha^{\tau}$ so that $\alpha^{5} \in K_{2}$ and $\alpha$ is uniquely determined up to a factor $\gamma$ of $K_{2}$. Thus we can take $\alpha$ to be an integer of $K_{2}$ and so $a$ and $e$ will be integers of $k_{3}$. Theorem 1 of Parry [7] shows that the only units of $K_{2}$ are the units of $k_{3}$, so if $\alpha^{5}$ were a unit of $K_{2}$, then $\alpha^{5} \in K_{1}$. This would mean that $M=K_{1}(\alpha)=K_{1}\left(\sqrt[5]{\alpha^{5}}\right)$ and so $M / K_{1}$ would be a nonnormal extension. Thus, $\alpha^{5}$ is not a unit of $K_{2}$.

If $5 \mid h_{1}$, then we may assume $M / K_{1}$ is unramified; and hence, $M(\zeta) / L$ is also unramified. Because $M(\zeta)=L\left(\sqrt[5]{\alpha^{5}}\right)$, a prime ideal $\mathfrak{B}$ of $L$ can divide $\left(\alpha^{5}\right)$ if and only if $\mathfrak{B}^{5}$ divides $\left(\alpha^{5}\right)$. Since $\alpha^{5} \in K_{2}$, a prime ideal $\mathfrak{p}$ of $K_{2}$ will divide $\left(\alpha^{5}\right)$ if and only if $\mathfrak{p}^{5}$ divides $\left(\alpha^{5}\right)$. Since we may assume $\alpha^{5}$ is not divisible by a fifth power of another integer of $K_{2}$ (except units), it follows $\left(\alpha^{5}\right)=\left(p_{1} \cdots p_{t}\right)^{5}$ where $\mathfrak{p}_{1} \cdots \mathfrak{p}_{t}$ is a nonprincipal ideal of $K_{2}$ whose fifth power is principal. Thus, 5 divides $h_{2}$.

THEOREM 5 (MAIN Result). If $5 \mid h_{2}$, then either $5 \mid h_{1}$ or the fundamental units $\epsilon_{1}=(a+b \sqrt{m}) / 2$ of $k_{1}$ and $\epsilon_{2}=(c+d \sqrt{5 m}) / 2$ of $k_{2}$ satisfy one of the following conditions:

(1) $a \equiv 0$ or $b \equiv 0(\bmod 25)$.

(2) $m \equiv \pm 2(\bmod 5)$ and $\epsilon_{1} \equiv \pm \epsilon$ or $\pm 7 \epsilon(\bmod 25)$ where $\epsilon=r \pm m^{2} \sqrt{m}$ with $r=9$ or 12 according as $m \equiv 2$ or $-2(\bmod 5)$.

(3) $d \equiv 0(\bmod 5)$.

Conversely, if $5 \mid h_{1}$ or one of conditions (1)-(3) holds, then $5 \mid h_{2}$.

Proof. We begin by reversing the roles of $K_{1}$ and $K_{2}$ in the proof of the preceding lemma. Thus, if $5 \mid h_{2}$, then $M / K_{2}$ is an abelian unramified extension of degree 5 and $M(\zeta)=L(\alpha)$ with $\alpha^{5} \in K_{1}$. If $\alpha^{5}$ is not a unit of $K_{1}$, then it follows as in Lemma 4 that $5 \mid h_{1}$. If $\alpha^{5}=e$ is a unit of $K_{1}$, then $\alpha$ may be replaced with $\alpha^{2}$ so that $\alpha^{5}=e=e_{1} e_{2} e_{3}$ with $e_{i} \in k_{i}(i=1,2,3)$ (see Theorems 1 and 2). Satz 119 of Hecke [2] shows that $L(\sqrt[5]{e}) / L$; and hence, $M / K_{2}$ will be an unramified extension if and only if

$$
x^{5} \equiv e \bmod (1-\zeta)^{5}
$$

is solvable in $L$. By applying the relative norm function for $L / K_{1}$, it is seen that (4) is solvable if and only if

$$
x^{5} \equiv e \bmod (5 \sqrt{5})
$$

is solvable in $K_{1}$. Applying the relative norm functions for $K_{1} / k_{i}(i=1,2,3)$ to (5) shows that

$$
\begin{array}{ll}
x^{5} \equiv e_{1} & \bmod (25) \\
x^{5} \equiv e_{2} & \bmod \mathfrak{p}_{5}^{3} \\
x^{5} \equiv e_{3} & \bmod (5 \sqrt{5})
\end{array}
$$

(where $\mathfrak{p}_{5}=(5, \sqrt{5 m})$ ) must be solvable in $k_{1}, k_{2}$ and $k_{3}$, respectively. First of all, it is easy to see that (8) has no solution unless $e_{3}$ is the fifth power of a unit of $k_{3}$. Thus, we may take $e_{3}=1$ and $\alpha^{5}=e=e_{1} e_{2}$. Next observe (7) is solvable if and 
only if $e_{2} \equiv u+v \sqrt{5 m}(\bmod 5)$ with $v \equiv 0(\bmod 5)$. Suppose $e_{2}=\epsilon_{2}^{t}$ where $\epsilon_{2}$ is the fundamental unit of $k_{2}$. Certainly, we may assume that $t$ is reduced modulo 5 . Moreover, if $t \neq \equiv(\bmod 5)$, then (7) has a solution if and only if

$$
x^{5} \equiv \epsilon_{2} \quad \bmod \mathfrak{p}_{5}^{3}
$$

has a solution; i.e. we may assume $t=0$ or 1 . If $t=1$, then condition (3) of the theorem holds. If $t=0$, then $e_{1} \neq \pm 1$, since otherwise $\alpha$ would be a 10th root of unity. Hence, we may assume that (6) holds where $e_{1}=\epsilon_{1}$ is the fundamental unit of $k_{1}$.

We need to determine exactly when

$$
x^{5} \equiv \epsilon_{1} \quad(\bmod 25)
$$

has a solution in $k_{1}$.

If $m \equiv \pm 1(\bmod 5)$, then $(25)=\left(\mathfrak{p}_{1} \mathfrak{p}_{2}\right)^{2}$ in $k_{1}$ where $\mathfrak{p}_{1}$ and $\mathfrak{p}_{2}$ are distinct prime ideals. Now (9) has a solution if and only if

$$
x^{5} \equiv \epsilon_{1} \quad\left(\bmod \mathfrak{p}_{i}^{2}\right)
$$

has a solution for $i=1,2$. Also, the reduced residue system modulo 25 forms a reduced residue system modulo $\mathfrak{p}_{i}^{2}$; and the fifth powers modulo $\mathfrak{p}_{i}^{2}$ are precisely \pm 1 and \pm 7 . If $\epsilon_{1} \equiv u+v \sqrt{m}(\bmod 25)$, then $\pm 1 \equiv u^{2}-m v^{2}(\bmod 25)$; and since $m \equiv \pm 1(\bmod 5)$, either $u \equiv 0$ or $v \equiv 0(\bmod 5)$. It follows that $u^{2} \equiv \pm 1$ or $m v^{2} \equiv$ $\pm 1(\bmod 25)$, and thus $u \equiv \pm 1, \pm 7$ or $\sqrt{m v} \equiv \pm 1, \pm 7\left(\bmod p_{i}^{2}\right)$. Suppose

$$
\epsilon_{1} \equiv u+v \sqrt{m}\left(\bmod \mathfrak{p}_{i}^{2}\right)
$$

where $v \equiv 0(\bmod 5)$. Thus, both $\epsilon_{1}$ and $u$ are fifth power residues and $v \equiv 0\left(\bmod \mathfrak{p}_{i}\right)$. It follows that

$$
\epsilon_{1} \equiv u \quad\left(\bmod p_{i}^{2}\right)
$$

and so $v \sqrt{m} \equiv 0 \bmod \mathfrak{p}_{i}^{2}$ which implies $v \equiv 0(\bmod 25)$. A similar argument shows that $u \equiv 0(\bmod 25)$ when $u \equiv 0(\bmod 5)$.

If $m \equiv \pm 2(\bmod 5)$, then 5 stays prime in $k_{1}$; and there are 600 reduced residues modulo 25, 24 of which are fifth powers. A complete set of fifth power residues may be obtained by taking all products from the sets

$$
S=\left\{ \pm 1, \pm 7, \pm m^{2} \sqrt{m}, \pm 7 m^{2} \sqrt{m}\right\} \text { and } T=\left\{ \pm 1, r \pm m^{2} \sqrt{m}\right\}
$$

where $r=9$ or 12 according as $m \equiv 2$ or $m \equiv 3(\bmod 5)$. Note that $r^{2}-m^{5} \equiv 1$ or $-1(\bmod 25)$ according as $m \equiv 3$ or $m \equiv 2(\bmod 5)$. Thus, only \pm 1 and \pm 7 times $r \pm m \sqrt[2]{m}$ can be units. It is now obvious that (9) has a solution if and only if (1) or (2) holds.

We have now proved that if $5 \mid h_{2}$ and $5 \nmid h_{1}$, then one of (1)-(3) must hold. Conversely, if one of (1)-(3) holds, set $e=\epsilon_{1}$ if (1) or (2) holds and $e=\epsilon_{2}$ if (3) holds. The above discussion shows that (4) has a solution for this choice of $e$. Satz 119 of Hecke [2] shows that $L(\sqrt[5]{e}) / L$ is unramified so that $5 \mid h$. Theorem 1 shows $5 \mid h_{1}$ or $5 \mid h_{2}$. If $5 \mid h_{1}$, then Lemma 4 shows $5 \mid h_{2}$, also. 
The following corollary gives a more convenient version of condition (2).

Corollary 6. The fundamental unit $\epsilon_{1}$ of $k_{1}$ satisfies condition (2) if and only if $\operatorname{Tr}\left(\epsilon_{1}\right) \equiv \pm 1, \pm 7(\bmod 25)$ where $\operatorname{Tr}$ denotes the trace function.

Proof. Certainly, if $\epsilon_{1}$ satisfies condition (2), then $\operatorname{Tr}\left(\epsilon_{1}\right) \equiv \pm 1, \pm 7(\bmod 25)$. Conversely, suppose $\epsilon=\epsilon_{1} \equiv a+b \sqrt{m}(\bmod 25)$ with $\operatorname{Tr}(\epsilon) \equiv 2 a \equiv \pm 1, \pm 7(\bmod$ 25). Thus,

$$
\pm 1 \equiv N(\epsilon) \equiv a^{2}-b^{2} m \quad(\bmod 25)
$$

so

$$
\begin{aligned}
\pm 4 & \equiv 4 a^{2}-4 b^{2} m \equiv \operatorname{Tr}(\epsilon)^{2}-4 b^{2} m \\
& \equiv \pm 1-4 b^{2} m \quad(\bmod 25) .
\end{aligned}
$$

Since $m \neq 0(\bmod 5)$, the choice of \pm signs must be the same on both sides and, in fact, is the sign of $\operatorname{Tr}(\epsilon)^{2}$. Thus,

$$
4 b^{2} m \equiv-3 \operatorname{Tr}(\epsilon)^{2} \quad(\bmod 25),
$$

so

$$
b^{2} m \equiv 18 \operatorname{Tr}(\epsilon)^{2} \equiv-7 \operatorname{Tr}(\epsilon)^{2} \quad(\bmod 25)
$$

Squaring gives

$$
b^{4} m^{2} \equiv-1 \quad(\bmod 25)
$$

so

$$
b \equiv-b^{5} m^{2} \quad(\bmod 25)
$$

Now

$$
b^{2} m \equiv-7 \operatorname{Tr}(\epsilon)^{2} \equiv-2 \operatorname{Tr}(\epsilon)^{2} \quad(\bmod 5)
$$

so

$$
b^{2} \equiv \pm \operatorname{Tr}(\epsilon)^{2} \quad(\bmod 5),
$$

where the sign is + if $m \equiv 3(\bmod 5)$ and - if $m \equiv 2(\bmod 5)$. If $m \equiv 3(\bmod 5)$, then

$$
b \equiv \pm \operatorname{Tr}(\epsilon)(\bmod 5)
$$

so

$$
b \equiv-b^{5} m^{2} \equiv \pm \operatorname{Tr}(\epsilon) m^{2} \quad(\bmod 25)
$$

Thus,

$$
\begin{aligned}
\epsilon & \equiv a \pm \operatorname{Tr}(\epsilon) m^{2} \sqrt{m} \quad(\bmod 25) \\
& \equiv-\operatorname{Tr}(\epsilon)\left(12 \pm m^{2} \sqrt{m}\right) \quad(\bmod 25)
\end{aligned}
$$


If $m \equiv 2(\bmod 5)$, then

$$
b^{2} \equiv-\operatorname{Tr}(\epsilon)^{2} \quad(\bmod 5)
$$

so

$$
b \equiv \pm 7 \operatorname{Tr}(\epsilon) \quad(\bmod 5) .
$$

Hence,

$$
b \equiv-b^{5} m^{2} \equiv \pm 7 \operatorname{Tr}(\epsilon) m^{2} \quad(\bmod 25),
$$

so

$$
\begin{aligned}
\epsilon & \equiv 13 \operatorname{Tr}(\epsilon) \pm 7 \operatorname{Tr}(\epsilon) m^{2} \sqrt{m} \quad(\bmod 25) \\
& \equiv-\operatorname{Tr}(\epsilon)\left(12 \pm 7 m^{2} \sqrt{m}\right) \quad(\bmod 25) \\
& \equiv \pm 7 \operatorname{Tr}(\epsilon)\left(9 \pm m^{2} \sqrt{m}\right) \quad(\bmod 25)
\end{aligned}
$$

Thus, in either case (2) is satisfied.

The distinction between conditions (1) and (2) of Theorem 5 is somewhat artificial as is seen by the following result.

Corollary 7. If $\epsilon_{1}$ satisfies condition (2), then $\epsilon_{1}^{3}$ satisfies condition (1).

Proof. Simply cube $\epsilon=r \pm m^{2} \sqrt{m}$ and note that $m^{5} \equiv 7$ or -7 and $r \equiv 9$ or $12(\bmod 25)$ according as $m \equiv 2$ or $-2(\bmod 5)$.

We now classify those fields $K_{2}$ which have class number divisible by 5 into three types:

Type 1. Condition (1) or (2) of Theorem 5 is satisfied.

Type 2. Condition (3) of Theorem 5 is satisfied.

Type 3. 5 divides $h_{1}$.

Type 3 fields can be subdivided into two further types:

Type 3a. 5 divides $h_{1}^{*}$.

Type 3b. 5 divides $h_{2}^{*}$.

The next corollary gives the sought after condition for 5 to divide $h_{1}$.

Corollary 8. If $5 \mid h_{2}$ and $K_{2}$ is not of Type 1 or 2 , then $5 \mid h_{1}$.

Corollary 9. If $K_{2}$ is both Type 1 and Type 2 , then $25 \mid h_{2}$ and the 5-class group of $K_{2}$ is noncyclic.

Proof. Under our assumptions $L\left(\sqrt[5]{\epsilon_{1}}\right)$ and $L\left(\sqrt[5]{\epsilon_{2}}\right)$ are distinct unramified abelian extensions of $L$ of degree 5. There exist corresponding unramified abelian extensions $M_{1} / K_{2}$ and $M_{2} / K_{2}$ of degree 5 with $M_{i} \subset L\left(\sqrt[5]{\epsilon_{2}}\right)$ for $i=1,2$. Since $L\left(\sqrt[5]{\epsilon_{1}}\right)$ $\neq L\left(\sqrt[5]{\epsilon_{2}}\right)$ we see $M_{1} \neq M_{2}$. Thus, $M_{0}=M_{1} M_{2}$ is an unramified abelian extension of $K_{2}$ of degree 25 with noncyclic Galois group. Thus, $25 \mid h_{2}$ and the 5-class group of $K_{2}$ is noncyclic.

Corollary 10. If $K_{2}$ is of Type 1 and Type $3 \mathrm{~b}$ or Type 2 and Type 3a, then $25 \mid h_{2}$ and the 5-class group of $K_{2}$ is noncyclic.

Proof. If $K_{2}$ satisfies both Type 1 and Type 2 conditions, then we are done by Corollary 9. When $K_{2}$ is of Type 3a (3b), there exists a nonprincipal prime ideal $p$ of 
$k_{1}\left(k_{2}\right)$ such that $p^{5}=(r+s \sqrt{m})$ is principal. (Here we temporarily change notation to allow $m \equiv 0(\bmod 5)$ when $K_{2}$ is Type 3b.) If we can choose $\alpha=r+s \sqrt{m}$ so that 5 does not ramify in $L(\sqrt[5]{\alpha})$, then we are done. This is so because $L(\sqrt[5]{\alpha}) / L$ and $L\left(\sqrt[5]{\epsilon_{i}}\right) / L\left(i=1\right.$ or 2 according as $K_{2}$ is Type $3 b$ or $\left.3 a\right)$ will be distinct unramified abelian extensions of degree 5. At this point, we can use the proof of Corollary 9.

In order to see that $\alpha$ can be chosen properly, it will be necessary to consider three cases:

Case $1 . K_{2}$ Type 2 and Type $3 a, m \equiv \pm 1(\bmod 5)$. Here $(25)=\left(p_{1} p_{2}\right)^{2}$ where $\mathfrak{p}_{1}$ and $\mathfrak{p}_{2}$ are prime ideals of $k_{1}$. There are 20 reduced residues modulo $\mathfrak{p}_{i}^{2}$ and the fifth powers are precisely $\pm 1, \pm 7$. Since $\epsilon_{1}$ is not a fifth power residue, the powers $\epsilon_{1}^{j}(j=0, \ldots, 4)$ form a complete set of coset representatives for the subgroup of fifth power residues in the whole group modulo $\mathfrak{p}_{i}^{2}$. Thus, $\epsilon_{\mathrm{I}}^{j}(r+s \sqrt{m})$ is a fifth power residue modulo $\mathfrak{p}_{i}^{2}$ for some $j$. We need to observe that $j$ does not depend on $i$. If

$$
\epsilon_{1}^{j}(r+s \sqrt{m}) \equiv u+v \sqrt{m}(\bmod 25)
$$

then as in the proof of Theorem 5 we must have $u \equiv 0$ or $v \equiv 0(\bmod 25)$. Thus, $\alpha=\epsilon_{1}^{j}(r+s \sqrt{m})$ is a fifth power modulo 25 and Satz 119 of Hecke [2] shows $L(\sqrt[5]{\alpha}) / L$ is an unramified extension.

Case 2. $K_{2}$ Type 2 and Type $3 \mathrm{a}, m \equiv \pm 2(\bmod 5)$. Here $L(\sqrt[5]{\alpha}) / L$ will be unramified if $\alpha$ is a fifth power residue modulo 25 . Since 5 remains prime in $k_{1}$, there are 600 reduced residues in $k_{1}$ modulo 25 and 24 of these are fifth power residues. If $A$ denotes the ring of algebraic integers of $k_{1}$, then the norm function defines a surjective homomorphism

$$
N:(A / 25 A)^{*} \rightarrow(Z / 25 Z)^{*} .
$$

The kernel of $N$ must have order 30 and the preimage, $H$, of $\{ \pm 1, \pm 7\}$ has order 120 . Note that $\epsilon_{1}, \alpha$ and the subgroup, $F$, of fifth power residues all belong to $H$. Since $\epsilon_{1}$ is not in $F$, the powers $\epsilon_{1}^{j}(j=0, \ldots, 4)$ give a complete set of coset representatives for $F$ in $H$. Thus, $\epsilon^{j} \alpha \in F$ for some choice of $j$. If $\alpha$ is replaced by $\epsilon_{1}^{j} \alpha$, then $L(\sqrt[5]{\alpha}) / L$ will be unramified.

Case 3. $K_{2}$ Type 1 and Type $3 \mathrm{~b}, m \equiv 0(\bmod 5)$. We shall now return to our standard notation and write $\alpha=r+s \sqrt{5 m}$ with $(m, 5)=1$. Now $L(\sqrt[5]{\alpha}) / L$ will be unramified if and only if $\alpha$ is a fifth power residue modulo $p_{5}^{3}$ where $p_{5}=(5, \sqrt{5 m})$. There are 100 reduced residues modulo $p_{5}^{3}$, and the subgroup of fifth power residues is $F=\{ \pm 1, \pm 7\}$. If $A$ denotes the ring of algebraic integers of $k_{2}$, then the norm function defines a homomorphism

$$
N:\left(A / p_{5}^{3}\right)^{*} \rightarrow(Z / 25 Z)^{*}
$$

Since only integers congruent to $\pm 1(\bmod 5)$ can be norms, the image of $N$ has order 10. The kernel of $N$ must also have order 10 and the preimage, $H$, of $\langle \pm 1\rangle$ has order 20. Note that $\epsilon_{2}, \alpha$ and $F$ all belong to $H$. Since $\epsilon_{2} \notin F$ we have, as in Case $2, \epsilon_{2}^{j} \alpha$ $\in F$ for some $j$. This completes the proof. 
TABLE I $(m=p)$

\begin{tabular}{|c|c|c|c|c|c|c|c|}
\hline D & $\underline{h}_{2-}$ & $h_{2}^{*}$ & type & D & $\mathrm{h}_{2}$ & $\mathrm{~h}_{2-}^{*}$ & type \\
\hline 37 & 10 & & 2 & 1429 & 180 & 2 & $3 a$ \\
\hline 53 & 10 & & 1 & 1493 & 250 & 18 & 1,2 \\
\hline 73 & 10 & & 1 & 1597 & 250 & 2 & 1,2 \\
\hline 89 & 20 & & 1 & 1621 & 320 & & 1 \\
\hline 92 & 20 & & 2 & 1637 & 450 & 14 & 1 \\
\hline 109 & 20 & & 1 & 1721 & 400 & 4 & 2 \\
\hline 124 & 40 & & 2 & 1741 & 400 & 4 & 1 \\
\hline 149 & 20 & & 2 & 1756 & 320 & 2 & $3 a$ \\
\hline 236 & 80 & & 2 & 1777 & 370 & & 1 \\
\hline 241 & 40 & & 1 & 1861 & 320 & & 1 \\
\hline 257 & 50 & & 1,2 & 1868 & 500 & 10 & $1,3 b$ \\
\hline 281 & 40 & & 2 & 1913 & 250 & 2 & 1,2 \\
\hline 293 & 50 & & 2 & 1916 & 320 & 2 & 2 \\
\hline 313 & 50 & & 2 & 1949 & 260 & 6 & 2 \\
\hline 401 & 80 & & $3 a$ & 1973 & 370 & 2 & 2 \\
\hline 428 & 100 & & 1 & 1996 & 400 & 6 & $2,3 a$ \\
\hline 433 & 90 & & 1 & 2092 & 340 & 2 & 1 \\
\hline 457 & 50 & 2 & 1,2 & 2348 & 500 & 2 & 1,2 \\
\hline 508 & 100 & & 1,2 & 2524 & 520 & 2 & 2 \\
\hline 509 & 100 & 4 & 1,2 & 2572 & 500 & 2 & 2 \\
\hline 541 & 80 & & 1 & 2732 & 740 & 2 & 2 \\
\hline 556 & 80 & & 1 & 2876 & 640 & & 1 \\
\hline 557 & 130 & 2 & 2 & 2908 & 740 & 2 & $3 a$ \\
\hline 617 & 130 & & 1 & 2972 & 580 & 2 & 2 \\
\hline 673 & 90 & & 1 & 3356 & 1280 & 2 & 2 \\
\hline 761 & 80 & 4 & 1 & 3548 & 740 & 2 & 2 \\
\hline 764 & 200 & & 1,2 & 3644 & 1000 & 10 & $3 b$ \\
\hline 796 & 160 & & 2 & 3788 & 900 & 2 & 1 \\
\hline 809 & 100 & 4 & 2 & 3932 & 1220 & & 1 \\
\hline 844 & 200 & & 1 & 4124 & 680 & & 1 \\
\hline 857 & 170 & & 1 & 4204 & 680 & & 1 \\
\hline 881 & 200 & 2 & 1,2 & 4252 & 820 & 2 & 2 \\
\hline 892 & 260 & & 2 & 4348 & 1220 & & $i$ \\
\hline 908 & 180 & & 2 & 4492 & 1780 & 10 & $2,3 b$ \\
\hline 937 & 130 & 2 & 2 & 4748 & 900 & 2 & 1 \\
\hline 997 & 130 & 2 & 2 & 4924 & 1000 & 2 & 1,2 \\
\hline 1069 & 100 & 2 & 2 & 5116 & 1600 & 10 & $1,3 \mathrm{~b}$ \\
\hline 1084 & 200 & & 1 & 5164 & 1960 & 2 & 2 \\
\hline 1093 & 250 & 2 & $3 a$ & 5308 & 900 & 2 & $2,3 a$ \\
\hline 1097 & 170 & 2 & 2 & 5708 & 1220 & 2 & 1 \\
\hline 1129 & 180 & 2 & 2 & 5804 & 1000 & 2 & 2 \\
\hline 1193 & 290 & 2 & 2 & 5932 & 1220 & 6 & 2 \\
\hline 1213 & 250 & 2 & 1 & 6044 & 1640 & 2 & 2 \\
\hline 1217 & 170 & 10 & $3 b$ & 6124 & 1000 & 6 & 1,2 \\
\hline 1228 & 260 & & 2 & 6284 & 1640 & 2 & 2 \\
\hline 1289 & 180 & & 1 & 6316 & 1360 & 2 & 2 \\
\hline 1301 & 200 & 2 & 1 & 6652 & 1940 & & 1 \\
\hline 1321 & 360 & & 1 & 6796 & 2320 & & 1 \\
\hline 1388 & 180 & & 2 & 6892 & 1780 & & 1 \\
\hline \multirow[t]{6}{*}{1428} & 180 & & 2 & 7132 & 2340 & 2 & 2 \\
\hline & & & & 7388 & 1300 & 10 & $3 b$ \\
\hline & & & & 7628 & 1700 & 2 & 1,2 \\
\hline & & & & 7916 & 1360 & 10 & $3 b$ \\
\hline & & & & 7996 & 1600 & 6 & 1,2 \\
\hline & & & & 8012 & 2900 & 2 & 1,2 \\
\hline
\end{tabular}


TABLE II $(m=2 p)$

\begin{tabular}{|c|c|c|c|c|c|c|c|}
\hline m & $h_{2}$ & $\mathrm{~h}_{2}^{*}$ & type & m & $\mathrm{h}_{2}$ & $\mathbf{h}_{2}^{*}$ & type \\
\hline 14 & 20 & & 2 & 1294 & 1300 & 2 & 2 \\
\hline 26 & 20 & & 2 & 1354 & 1220 & 12 & 2 \\
\hline 38 & 40 & & 1 & 1366 & 900 & 2 & 1,2 \\
\hline 62 & 40 & & 1 & 1382 & 1040 & & 1 \\
\hline 82 & 80 & & 1 & 1402 & 1960 & & $i$ \\
\hline 86 & 100 & & 2 & 1466 & 1620 & 20 & $3 b$ \\
\hline 134 & 100 & & 1 & 1478 & 1640 & 2 & ? \\
\hline 202 & 200 & & 1 & 1486 & 1460 & 2 & $3 a$ \\
\hline 214 & 260 & & 1 & 1514 & 900 & 20 & $3 b$ \\
\hline 278 & 360 & & 1 & 1546 & 820 & 4 & 2 \\
\hline 298 & 400 & & 1,2 & 1654 & 1300 & 2 & 2 \\
\hline 314 & 260 & & 1 & 1658 & 1040 & & 1 \\
\hline 326 & 260 & & 1 & 1754 & 2340 & & 1 \\
\hline 358 & 200 & & 2 & 1762 & 1360 & & 1 \\
\hline 382 & 360 & & 1 & 1766 & 1700 & 2 & $3 \mathbf{a}$ \\
\hline 398 & 320 & & 2 & 1838 & 2080 & & 1 \\
\hline 422 & 400 & 2 & 1 & 1874 & 2340 & & 1 \\
\hline 446 & 340 & 6 & 2 & 1882 & 1960 & 20 & $3 b$ \\
\hline 458 & 320 & 4 & 2 & 1934 & 1700 & 10 & $3 b$ \\
\hline 466 & 580 & 4 & 1 & 1954 & 1460 & 4 & 2 \\
\hline 502 & 400 & 6 & 1,2 & 1966 & 1300 & 6 & 1 \\
\hline 514 & 340 & & 1 & 1982 & 1160 & & \\
\hline 526 & 500 & 2 & 1,2 & & & & \\
\hline 554 & 740 & 4 & 2 & & & & \\
\hline 622 & 520 & & 1 & & & & \\
\hline 626 & 500 & 4 & 1,2 & & & & \\
\hline 634 & 340 & & 1 & & & & \\
\hline 662 & 400 & 2 & 1 & & & & \\
\hline 674 & 580 & & 1 & & & & \\
\hline 734 & 500 & 6 & 1,2 & & & & \\
\hline 758 & 520 & 2 & 2 & & & & \\
\hline 766 & 500 & 2 & 2 & & & & \\
\hline 794 & 740 & 20 & $3 b$ & & & & \\
\hline 842 & 520 & 4 & 2 & & & & \\
\hline 922 & 1000 & 4 & 1,2 & & & & \\
\hline 926 & 740 & 2 & 2 & & & & \\
\hline 982 & 1040 & 6 & $1,3 a$ & & & & \\
\hline 1006 & 1220 & 2 & 2 & & & & \\
\hline 1018 & 640 & 4 & 2 & & & & \\
\hline 1042 & 800 & 8 & 1 & & & & \\
\hline 1114 & 1460 & 4 & 2 & & & & \\
\hline 1126 & 900 & 10 & $2,3 a, 3 b$ & & & & \\
\hline 1142 & 1360 & 2 & 2 & & & & \\
\hline 1198 & 800 & 2 & 2 & & & & \\
\hline 1214 & 1220 & & 1 & & & & \\
\hline 1226 & 1460 & 4 & $3 a$ & & & & \\
\hline 1238 & 1000 & 2 & 1,2 & & & & \\
\hline 1262 & 1160 & & 1 & & & & \\
\hline
\end{tabular}

It is interesting to note that when $m=982, K_{2}$ is of Types 1 and $3 \mathrm{a}$ and when $m=1123, K_{2}$ is of Types 2 and $3 \mathrm{~b}$. However, 25 does not divide $h_{2}$ in either case!

Corollary 11. If $K_{2}$ is of both Type 3a and Type 3b, then $25 \mid h_{2}$ and the 5-class group of $K_{2}$ is noncyclic.

Proof. Corollary 10 shows that we may assume that $K_{2}$ is of neither Type 1 nor Type 2. Thus, as in the proof of that corollary, we may choose $\alpha_{i} \in k_{i}$ such that $L\left(\sqrt[5]{\alpha_{i}}\right) / L(i=1,2)$ is an unramified abelian extension of degree 5. Moreover, we 
may assume $\left(\alpha_{i}\right)=\mathfrak{p}_{i}^{5}$ where $\mathfrak{p}_{i}$ is a nonprincipal prime ideal of $k_{i}(i=1,2)$. If $L\left(\sqrt[5]{\alpha_{1}}\right)=L\left(\sqrt[5]{\alpha_{2}}\right)$, then $\alpha_{1}=\beta^{5} \alpha_{2}^{t}$ for some $\beta \in K_{1}$ and $t=1,2,3$ or 4 . Applying the norm function for $K_{1} / k_{1}$ gives $\alpha_{1}^{2}=\left(N(\beta) p_{2}^{t}\right)^{5}$, where $p_{2}$ is a prime integer. Since $L\left(\sqrt[5]{\alpha_{1}}\right) / L$ is of degree 5 , we must have $L\left(\sqrt[5]{\alpha_{1}}\right) \neq L\left(\sqrt[5]{\alpha_{2}}\right)$. The proof of Corollary 9 now applies.

COROLlary 12. Let $K_{2}$ be of Type $i(i=1$ or 2$), \epsilon=\epsilon_{i}$ and $\theta=\sqrt[5]{\epsilon}+\sqrt[5]{\epsilon^{\prime}}$, where $\epsilon^{\prime}$ denotes the conjugate of $\epsilon$ and both fifth roots are real. Then $M=K_{2}(\theta)$ is an unramified abelian extension of $K_{2}$ of degree 5 and $\theta$ is a root of

$$
f(x)=x^{5}-5 N(\epsilon) x^{3}+5 x-\operatorname{Tr}(\epsilon),
$$

where $N(\epsilon)$ and $\operatorname{Tr}(\epsilon)$ denote the norm and trace of $\epsilon$.

Proof. Merely reverse the roles of $K_{1}$ and $K_{2}$ in the proof of Lemma 4. Under our assumptions we can take $\alpha=\sqrt[5]{\epsilon}$ and $\alpha^{\rho}=\sqrt[5]{\epsilon^{\prime}}$. It is easy to see $a=N(\epsilon)$ and $a e$ $=\operatorname{Tr}(\epsilon)$.

5. Numerical Results. Since $K_{2}$ is an imaginary cyclic biquadratic field, its class number can be readily computed using a result of Hasse [1]. The formula is

$$
h_{2}=\frac{1}{2 \mathfrak{f}^{2}}\left|\sum_{n(\bmod f)} \chi(n) n\right|^{2},
$$

where $f$ is the conductor of $K_{2}$, the summation is over the smallest reduced residue system modulo $f$ and $\chi(n)=(m / n) \chi_{1}(n)$. Here $(m / n)$ is the Jacobi symbol and $\chi_{1}(n)$ is a primitive character modulo 5 defined by $\chi_{1}(2)=i=\sqrt{-1}$. The conductor $\mathrm{f}=5 D$ where $D$ is the discriminant of $k_{1}$. When $\mathrm{f}$ is even, we can make the following simplification:

THEOREM 13. If $f$ is even, then

$$
h_{2}=\frac{1}{8}\left|\sum_{n(\bmod f / 2)} \chi(n)\right|^{2}
$$

Proof. Note that

$$
\chi(n+f / 2)=\left(\frac{m}{n+f / 2}\right) \chi_{1}(n+f / 2)=\left(\frac{m}{n+f / 2}\right) \chi_{1}(n),
$$

since $\mathrm{f} / 2=10 m$. Now either $m$ is odd or $m=2 r$ with $r$ odd. In the first case $m \equiv$ $3(\bmod 4)$ and in both cases $n$ is odd. In the former case

$$
\begin{aligned}
\left(\frac{m}{10 m+n}\right) & =(-1)^{((m-1) / 2)(10 m+n-1) / 2}\left(\frac{10 m+n}{m}\right) \\
& =(-1)^{(n+1) / 2}\left(\frac{n}{m}\right)=(-1)^{(n+1) / 2}(-1)^{(n-1) / 2}\left(\frac{m}{n}\right) \\
& =-\left(\frac{m}{n}\right) .
\end{aligned}
$$


In the second case

$$
\begin{aligned}
\left(\frac{m}{10 m+n}\right) & =\left(\frac{2 r}{20 r+n}\right)=\left(\frac{2}{20 r+n}\right)\left(\frac{r}{20 r+n}\right) \\
& =\left(\frac{2}{n+4}\right)\left(\frac{r}{n}\right)=-\left(\frac{2}{n}\right)\left(\frac{r}{n}\right)=-\left(\frac{2 r}{n}\right)=-\left(\frac{m}{n}\right) .
\end{aligned}
$$

In either case $\chi(n+f / 2)=-\chi(n)$ so

$$
\begin{aligned}
\sum_{n(\bmod f)} \chi(n) n & =\sum_{n(\bmod f / 2)} \chi(n) n+\chi(n+f / 2)(n+f / 2) \\
& =\sum_{n(\bmod f / 2)} \chi(n) n-\chi(n)(n+f / 2)=-f / 2 \sum_{n(\bmod f / 2)} \chi(n) .
\end{aligned}
$$

The desired result is now immediate.

Using FORTRAN programs, we have computed $h_{2}$ for all values of $m<2000$ where $m=p$ or $2 p$ with $p$ prime. In the tables above we list all such values of $m$ with 5 dividing $h_{2}$. The type (or types) of each field was determined using the table of Ince [4] and a program to compute $\epsilon_{2}$ (or $\epsilon_{2}$ modulo 100 when overflow occurred in double precision) when $5 m>2025$. If Corollary 10 did not show $\left(h_{2}^{*}, 5\right)=1$ and $m>405$, then $h_{2}^{*}$ was computed. This value appears in the tables whenever we computed it.

Department of Mathematics

Virginia Polytechnic Institute \& State University

Blacksburg, Virginia 24061

1. H. HASSE, Über die Klassenzahl abelscher Zahlkörper, Akademie-Verlag, Berlin, 1952. MR 14, 141 .

2. E. HECKE, Vorlesungen ̈̈̈ber die Theorie der algebraischen Zahlen, Leipzig, 1923.

3. C. S. HERZ, Construction of Class Fields, Lecture Notes in Math., vol. 21, SpringerVerlag, Berlin and New York, 1966. MR 34 \#1278.

4. E. L. INCE, Cycles of Reduced Ideals in Quadratic Fields, British Math. Assn. Tables, Vol. 4, London, 1934.

5. T. KUBOTA, “Über die Beziehung der Klassenzahlen der Unterkörper des bizyklischen biquadratischen Zahlkörpers,” Nagoya Math. J., v. 6, 1953, pp. 119-127. MR 15, 605.

6. S. KURODA, “Über den Dirichletschen Körper," J. Fac. Sci. Imp. Univ. Tokyo Sect. I, v. 4, 1943, pp. 383-406. MR 9, 12.

7. C. PARRY, "Units of algebraic numberfields," J. Number Theory, v. 7, 1975, pp. 385388. MR 52 \#5625.

8. C. WALTER, Class Number Relations in Algebraic Number Fields, Ph.D. Thesis, University of Cambridge, 1976.

9. P. WEINBERGER, "Real quadratic fields with class numbers divisible by $n$," $J$. Number Theory, v. 5, 1973, pp. 237-241. MR $49 \# 252$.

10. Y. YАMAMOTO, “On unramified galois extensions of quadratic number fields," Osaka $J$. Math., v. 7, 1970, pp. 57-76. MR 42 \#1800. 